\title{
Epidural catheter breakage
}

\author{
Fernandes M., Pereira E., Ferreira Silva L.I., Silva E. \\ Centro Hospitalar e Universitário de Coimbra, Departement of Anaesthesiology, Coimbra, Portugal
}

The epidural catheter is a widely used medical device. Anesthesiologists ordinarily place it in order to administer anesthesia and manage pain in several different clinical scenarios.

Although being considered a rather safe procedure, adverse events like dural punctures, spinal hematomas or epidural abscesses have been continuously reported since the introduction of this technique. The outcome of these complications can be devastating, with major neurological sequelae.

Among the different issues that the anesthesiologist may face while dealing with an epidural, breakage of the catheter is a rare but troublesome event. Entrapment around the spinal ligaments, kinking, knotting or looping of the catheter within the epidural space or subcutaneous tissues, defective devices, and a poor handling technique by the operator are among the potential causes of fracture.

The objective of this report was to describe a case where a retained epidural catheter fragment in labor analgesia was treated by surgery.

\section{Case Report}

- 35-year-old 우 caucasian

- Severe low back pain and paresthesia of the left lower limb, with progressive aggravation

- Symptoms started after labor analgesia with an epidural catheter. No mention of breakage had been made to her at that time, nor a retained foreign body was ever reported afterwards.

- Referred for neurosurgery: surgery extraction

- The patient underwent a balanced general anesthesia for two hours to fluoroscopy-guided extract the fragment of the epidural catheter, which was uneventful.

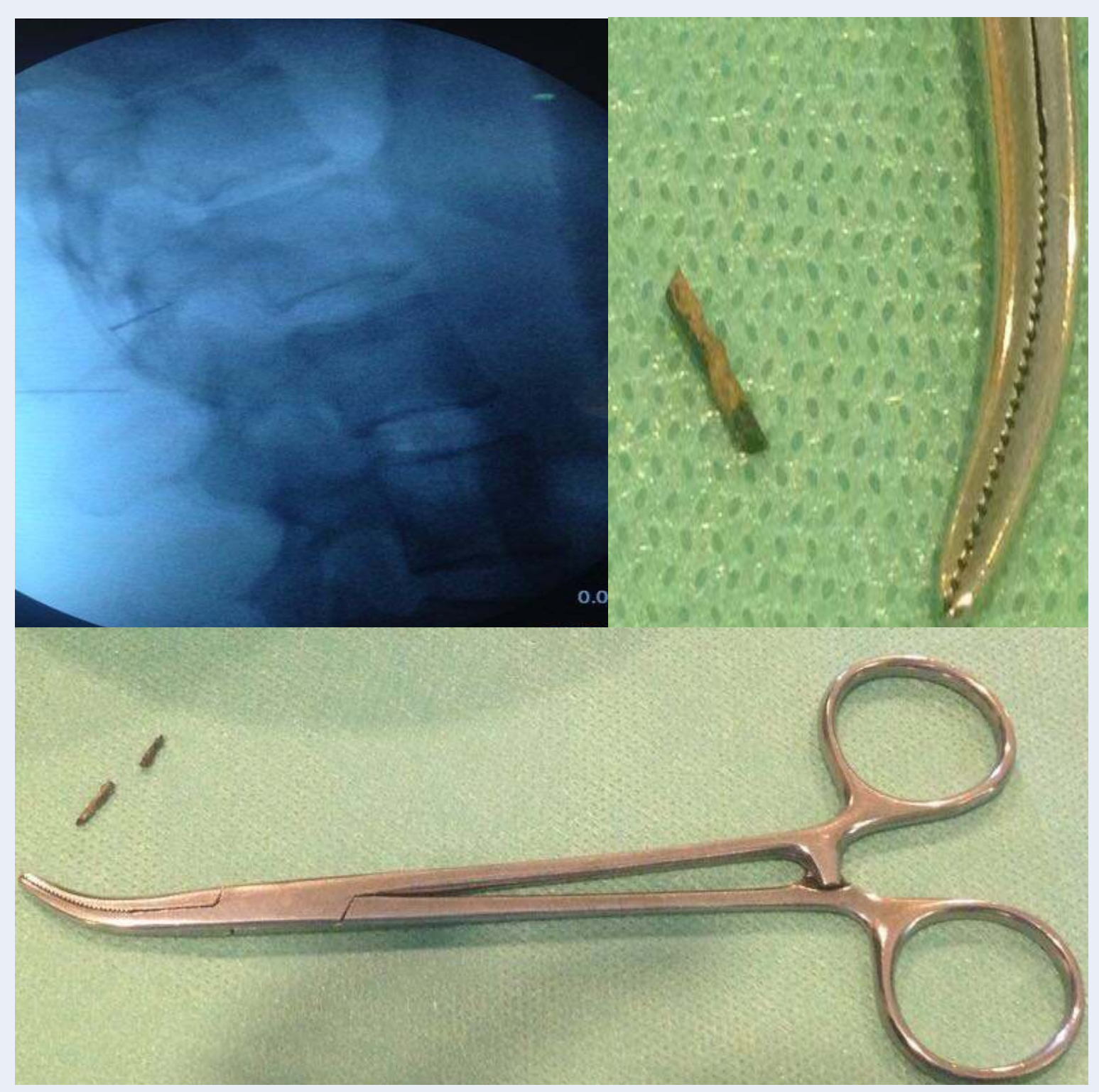

\section{Discussion}

Shearing of an epidural is a rare but serious adverse event, whose management may be problematic for the anesthesiologist. The greatest attention must be paid while handling the catheter, especially at its removal. Generally speaking, careful training of the healthcare personnel is probably the most effective preventive strategy. In addition, several technical options have been reported about what to do in case resistance is found during withdrawal of the catheter. When facing breakage of a catheter during removal the different authors agree that the treatment of small fragments in an asymptomatic patient should be conservative, informing the patient of the complication. 